African Crop Science Journal by African Crop Science Society is licensed under a Creative Commons Attribution 3.0 Uganda License. Based on a work at www.ajol.info/ and www.bioline.org.br/cs DOI: http://dx.doi.org/10.4314/acsj.v25i2.6

\title{
DETERMINANTS DE L'ADOPTION ET IMPACT DES VARIETES AMELIOREES SUR LA P RODUCTIVITE DU MIL AU NIGER
}

\author{
O.H. ISSOUFOU, S. BOUBACAR ${ }^{1}$, T. ADAM ${ }^{2}$ et B. YAMBA ${ }^{3}$ \\ Laboratoire de Productions Végétales, Faculté d'Agronomie, Université Abdou Moumouni de Niamey-Niger, \\ BP 10960 Niamey-Niger \\ ${ }^{1}$ Département de Sociologie et Economie Rurales, Faculté d'Agronomie, Université Abdou Moumouni de \\ Niamey-Niger, BP 10960 Niamey-Niger \\ ${ }^{2}$ Département de Productions Végétales, Faculté d'Agronomie, Université Abdou Moumouni de Niamey- \\ Niger, BP 10960 Niamey-Niger \\ ${ }^{3}$ Département de Géographie, Faculté des Lettres et des Sciences Humaines, Université Abdou Moumouni de \\ Niamey-Niger, BP 418 Niamey-Niger \\ Auteur de correspondance : isfouhaladou@yahoo.fr
}

(Received 2 January, 2017; accepted 10 May, 2017)

\section{RESUME}

Au Niger, l'adoption des nouvelles technologies par les producteurs est l'une des voies du développement de l'agriculture pluviale. L'étude analyse l'adoption et l'impact des variétés améliorées sur la productivité du mil, Pennisetum glaucum L.). Les déterminants de l'adoption ont été identifiés à l'aide d'un modèle Logit. Pour minimiser le biais lié à la différence entre les caractéristiques des adoptants et des non adoptants, la méthode de Local Average Response Function (LARF) basée sur l'approche contrefactuelle a été utilisée pour estimer l'effet moyen local du traitement « Local Average Treatment Effect » (LATE). Les données proviennent de 612 producteurs échantillonnés de manière aléatoire sur la base de la liste des producteurs au niveau de chacun des 15 villages d'étude. Les résultats donnent un taux d'adoption de 35,29\% des semences du mil amélioré. Cette adoption est significativement déterminée à $\mathrm{P}<0,001$ par la perception de risque de production, l'accès à la vulgarisation, la disponibilité et la productivité ; à $\mathrm{P}<0,01$ par l'adaptabilité, la précocité, l'âge, l'éducation et à $\mathrm{P}<0,05$ par la taille du ménage. Ces semences ont permis d'augmenter le rendement du mil de 406,93 $\mathrm{kg} \mathrm{ha}^{-1}$. On note une différence significative $(\mathrm{P}<0,05)$ entre les adoptants et les non adoptants sur le nombre de mois de consommation de leur production du mil. Ces déterminants d'adoption et l'impact sont des éléments à prendre en compte pour réussir les actions de diffusion des nouvelles technologies agricoles en milieu paysan.

Mots Clés : Adoption, Approche contrefactuelle, Variétés améliorées, Modèle Logit

\begin{abstract}
Niger is a country where adoption of new technologies by producers is one of the ways to develop rainfed agriculture. This study analyses the adoption and the impact of improved seeds varieties on millet (Pennisetum glaucum L.) productivity. The determinants of adoption were identified using a Logit model. To minimise bias in the characteristics of adopters and non-adopters, the Local Average Response Function (LARF) method based on the counterfactual approach was used to estimate the "Local Average Treatment Effect "(LATE). Data were collected from 612 producers choose by randomly sampled in their list in each of the 15 study villages. The results showed that millet seed adoption rate was $35.29 \%$. This adoption was significantly determined at $\mathrm{P}<0.001$ by perception of production risk, access to extension, availability and productivity; at $\mathrm{P}<0.01$ by
\end{abstract}


adaptability, early maturity, age, education and $\mathrm{P}<0.05$ by household size. In addition, millet yield increased to $406.93 \mathrm{~kg} \mathrm{ha}^{-1}$ through the adoption of the improved seed. There was also a significant difference $(\mathrm{P}<0,05)$ between adopters and non-adopters based on the number of months of consumption of their millet production. These determinants of adoption and the impact are factors to be taken into account in order to successfully disseminate new agricultural technologies in a farming environment.

Key Words: Adoption, Counterfactual approach, improved varieties, Logit model

\section{INTRODUCTION}

En Afrique de l'Ouest, l'augmentation de la productivité des facteurs de production est une composante essentielle du succès de toute stratégie de développement rural (Ngondjeb et al., 2014). En effet, dans cette région, l'agriculture représente près d'un quart du Produit Intérieur Brut (PIB) et emploie environ $70 \%$ de la population (Banque Mondiale, 2010). Cependant, malgré cette importance, l'activité n'a pas épargné 33,7 millions de personnes d'être en situation d'insécurité alimentaire [Food and Agriculture Organisation (FAO), 2015]. Ce nombre risque aussi d'augmenter à cause de la croissance démographique et du changement climatique qui constituent une lourde menace sur la production alimentaire. $\mathrm{Si}$ aucune mesure n'est prise pour renforcer la durabilité, la productivité et la résilience de l'agriculture, la situation risque de mettre en péril la réalisation des objectifs de développement durable consistant à éliminer la faim et la pauvreté à l'horizon 2030 (FAO, 2016).

Au Niger, plus de 2 millions de personnes sont en situation d'insécurité alimentaire [Institut National de la Statistique (INS) et Système d'Alerte Précoce et de prévention de Catastrophe (SAP/PC), 2016]. Cette sousalimentation est due en grande partie à la baisse de la productivité du mil qui représente $80 \%$ de la production nationale de céréales [(Institut de Recherche pour le Développement (IRD), 2004]. Le mil demeure la céréale de base de l'alimentation des populations et joue un rôle crucial dans la sécurité alimentaire au Niger. Avec un rendement moyen de $400 \mathrm{~kg} \mathrm{ha}^{-1}$, ce sont environ 2 millions de tonnes de graines de mil qui sont produites chaque année au
Niger sur une superficie de plus de 5 millions d'hectares (IRD, 2004). Ce niveau faible de productivité du mil est imputable à une faible adaptation des cultivars locaux entrainée par des contraintes biotiques, abiotiques et socioéconomiques (Agrhymet, 2012). Pour éviter le recours aux importations des céréales et à l'aide alimentaire, il devient nécessaire d'augmenter la productivité du mil au Niger. Les chercheurs et décideurs voient les semences des variétés améliorées comme un facteur indispensable à cette augmentation [Ministère du Développement Agricole (MDA), 2012 ; (FAO), 2016]. C'est ainsi que la recherche a créé des nouvelles variétés précoces, productives et résistante à la sécheresse et aux ravageurs. Ces variétés sont diffusées en milieu paysan par la vulgarisation agricole qui utilise des approches comme les champs écoles paysans, les groupes de contact, les formations et visites, les journées portes ouvertes et les conseils spécialisés. Dans toutes les régions agricoles du pays, des grands projets d'amélioration de la production agricole ont été mis en œuvre comme c'est le cas des distributions d'urgence de semences par l'Etat, le projet de multiplication et diffusion des semences de qualité des variétés améliorées et adaptées au changement climatique de la FAO, le Programme de Productivité Agricole en Afrique de l'Ouest (PPAAO) de la Banque Mondiale et bien d'autres. La recherche en économie de la production agricole a montré que les taux d'adoption et les facteurs influençant la décision des agriculteurs d'adopter une technologie agricole varient fortement entre les producteurs en raison de l'hétérogénéité de leurs préférences (Roussy et al., 2015). D'un autre côté, plusieurs études montrent que l'adoption des semences des 
variétés améliorées comme le riz, le blé et le niébé pourrait conduire à une augmentation de la production, une amélioration de la sécurité alimentaire et une augmentation du revenu des agriculteurs (Awotide et al., 2012; Arouna et Diagne, 2013 ; Shiferaw et al., 2014 ; Moti et al., 2015 ; Tesfaye et al., 2016). Cependant, la littérature disponible, surtout pour le cas du Niger, n'a pas encore abordé l'adoption et l'impact des semences améliorées de la culture du mil. Ainsi, nous contribuons à la littérature en analysant le niveau, les déterminants et l'impact de l'adoption des variétés améliorées sur la productivité du mil dans les régions de Tillabéri et Zinder au Niger avec des données d'enquête et une approche économétrique. Dans le cadre de cette étude, le modèle Logit est utilisé pour déterminer le niveau et déterminants d'adoption des semences des variétés améliorées du mil. Ce modèle est souvent employé dans l'analyse des décisions d'adoption des technologies agricoles non seulement pour des raisons de commodité mais aussi pour ses résultats probants et sa simplicité. Aussi, pour tenir compte des facteurs inobservables liés à l'identification et estimer les effets non biaisés du traitement sur le rendement du mil ou l'effet moyen local du traitement (LATE), l'étude a utilisé la méthode de Local Average Response Function (LARF) basée sur l'approche contrefactuelle. Cette méthodologie a fait l'objet de plusieurs applications et a montré ses preuves dans l'évaluation d'impact des technologies agricoles.

L'objectif de cette étude est d'analyser l'adoption et l'impact des semences des variétés améliorées sur la productivité du mil au Niger.

\section{METHODOLOGIE}

Zone d'étude. Les données de l'étude ont été collectées dans le Sud-Ouest dont le climat est de type tropical humide et le Centre-Est de climat de type sahélien du Niger. Les communes et villages d'étude ont été choisis avec l'aide des Agents de Service Départementaux de l'Agriculture sur la base des principaux critères suivants : (i) importance de la production du mil, (ii) bénéficiaires des semences améliorées du mil distribuées par l'Etat ou PPAAO ou FAO au cours des trois dernières années, iii) développement de l'activité de production de semences certifiées du mil et iv) existence d'au moins un privé qui appuie la diffusion des semences des variétés améliorées du mil. Ainsi, dans le Sud-Ouest, les villages d'étude sont : Balléyara, N'Gawa et Tabla (Ballkéyara), Boubon, Karma et Tagabatchi (Kollo), Mamaga, Bokki et Djabou (Say). Au niveau du Centre-Est, les villages enquêtés sont : Bougoum, Brundi, Tourmou et Kandine (Damagaram Takaya), Angoual Gamji et Bandé (Magaria). Au total 15 villages dont 9 dans la zone Sud-Ouest et 7 au Centre-Est ont été visités et enquêtés.

Collecte des données. Dans les zones agricoles du Niger, neuf variétés améliorées du mil, Pennisetum glaucum (L.) $R$. Br., sont utilisées par les agriculteurs. Ces variétés sont : H80-10GR, HKP, ICVM IS 89305, ICVM IS 94205, ICVM IS 94206, ICVM IS 99001, SOSSAT C88, Zatib et Zongo Kolo. La production de semences certifiées de première (R1) et deuxième (R2) génération s'élève à 5392 687,37 kg (MDA, 2016). Ces variétés ont été introduites par les institutions de recherches, les partenaires au développement, les services agricoles et les privés.

Pour mettre en évidence le niveau, déterminants et impact de l'adoption des semences des variétés améliorées sur la productivité du mil, la technique d'étude utilisée a combinée la méthode quantitative (questionnaire) à la méthode qualitative (entretiens informels et semi-structurés). Les personnes enquêtées sont les chefs de ménages agricoles. Ces personnes ont été échantillonnées sur la base de la liste des ménages agricoles de chaque village. Pour certains villages, cette liste a été élaborée lors du passage de la collecte de données. 
Par contre pour d'autres, nous avons utilisé la liste fournie par les Services de l'Agriculture. Dans ce dernier cas, une actualisation a été faite au village avec l'aide des chefs et autres personnes ressources. Pour l'ensemble des villages d'étude, 4159 ménages agricoles ont été recensés. Nous avons enquêté 612 ménages, correspondant à $15 \%$ du total.

Ces ménages enquêtés ont été choisis à l'aide de la méthode de tirage aléatoire simple sans remise après avoir numérotés tous les ménages de 1 à $\mathrm{N}$ pour chaque village. Les données ont été collectées à l'aide d'un questionnaire comportant des informations sur les caractéristiques socio-économiques, démographiques, l'utilisation des semences améliorées du mil, les perceptions sur le risque de production et sur les caractéristiques de ces semences améliorées, la production obtenue, le niveau des intrants utilisés, le nombre de mois de consommation de la production et les contraintes de production durant l'année culturale 2015-2016. L'entretien informel a été conduit sous forme d'interviews très ouvert avec les producteurs à chaque fois que l'occasion se présente.

Pour s'assurer de la véracité des informations recueillies, des observations et un croisement des informations fournies ont été réalisées. Les données secondaires recueillies dans ce cadre ont été confrontées aux données primaires.

\section{Analyse des données}

\section{Cadre analytique et techniques d'estimation}

Cadre économétrique du modèle Logit. Le producteur fonde sa décision d'adoption des variétés améliorées sur la base du principe de rationalité notamment l'hypothèse de maximisation de l'utilité de la théorie néoclassique. Ainsi, le producteur rationnel préfère la culture (variétés) qui lui procure le plus d'utilité.
Les travaux en économie sur l'adoption des technologies montrent que les décisions des agriculteurs sont influencées par des multiples facteurs endogènes ou exogènes (observables et non observables directement) et les caractéristiques intrinsèques de la technologie (Ngondjeb et al., 2011 ; Roussy et al., 2015).

Pour analyser l'adoption des variétés améliorées du mil, l'étude utilise le modèle Logit qui a montré ses preuves dans des études similaires (Roussy et al., 2015).

La fonction d'utilité d'un producteur i est donnée par :

$\mathrm{U}_{\mathrm{ij}}=\mathrm{U}_{\mathrm{ij}}$ (Mbetid-Bessane, 2014)

Considérant $\mathrm{X}_{\mathrm{i}}$ un vecteur colonne de $\mathrm{k}$ facteurs déterminant la décision d'adoption de la variété du mil et j l'utilité résultant du choix de la variété $(j=1,2)$. Le producteur choisira la variété 1 si $\mathrm{U}_{\mathrm{i} 1}>\mathrm{U}_{\mathrm{i} 2^{2}}$. Cette préférence de choix du producteur peut être représentée par la variable latente $Y_{i}^{*}$ telle que :

$Y_{i}^{*}=\beta X_{i}+\varepsilon_{i}$

$\beta$ est un vecteur ligne de $\mathrm{k}$ paramètres et $\varepsilon_{\mathrm{i}}$ une perturbation aléatoire; $\mathrm{Y}_{\mathrm{i}}^{*}>0$ si $\mathrm{U}_{\mathrm{i} 1}>\mathrm{U}_{\mathrm{i} 2}$; $\mathrm{Y}_{\mathrm{i}}^{*} \leq 0$ si $\mathrm{U}_{\mathrm{i} 1} \leq \mathrm{U}_{\mathrm{i} 2}$. En définissant une variable dichotomique $Y_{i}$ telle que $Y_{1}=1$ si la variété 1 du mil est choisie et $Y=0$ si non. La probabilité $\mathrm{P}_{\mathrm{i}} \mathrm{d}^{\prime}$ adoption de la variété 1 est donnée par :

$\mathrm{P}_{\mathrm{i}}=\operatorname{Prob}\left(\mathrm{Y}_{\mathrm{i}}=1\right)=\operatorname{Prob}\left(\mathrm{Y}_{1}{ }^{*}>0\right)=\operatorname{Prob}\left(\beta \mathrm{X}_{\mathrm{i}}+\right.$ $\left.\varepsilon_{\mathrm{i}}>0\right)=\operatorname{Prob}\left(\varepsilon_{\mathrm{i}}>-\beta^{\prime} \mathrm{X}_{\mathrm{i}}\right)$

En supposant une distribution symétrique de $\mathrm{C}_{\mathrm{i}}$, on obtient : $\mathrm{P}_{\mathrm{i}}=\operatorname{Prob}\left(\varepsilon_{\mathrm{i}}<\beta \mathrm{X}_{\mathrm{i}}\right)=\mathrm{F}\left(\beta \mathrm{X}_{\mathrm{i}}\right), \mathrm{F}$ est une fonction de répartition définie par la loi de $\left[_{i}\right.$. Selon que ${ }_{i}$ suit une loi normale ou une loi logistique, l'adoption de l'agriculteur peut être représentée par un modèle Logit ou Probit. La présente étude utilise le modèle Logit pour des raisons de simplicité. 
Selon Polome (2013), la forme standard du modèle Logit $\mathrm{G}$ est donnée par la formule :

$\mathrm{G}(\mathrm{z})=\exp (\mathrm{z}) /[1+\exp (\mathrm{z})]=\mathrm{A}(\mathrm{z})$

L'effet marginal de la variation de la probabilité d'adoption des semences améliorées suite à la variation d'une variable explicative est donné par :

$\mathrm{G}(\mathrm{z})=\exp (\mathrm{z}) /[1+\exp (\mathrm{z})]^{2} \quad$ (Polome, 2013)

A partir de la littérature théorique et économétrique sur l'adoption des technologies et des données d'enquête, les variables des modèles (Logit) ont été définies (Tableau 1). Dans le cadre de l'étude, la variable dépendante du modèle Logit est l'utilisation de semences améliorées du mil.

Une fois adoptées, la mesure de l'impact des variétés améliorées du mil peut se faire en utilisant le Local Average response Function (LARF).
Méthode de Local Average Response Function (LARF). Sur la base de la littérature, l'étude utilise les approches conventionnelle «naïve» et non-expérimentale dite « contrefactuelle» (Fall, 2006) pour mesurer l'impact des variétés améliorées du mil. L'approche naïve consiste à faire une simple comparaison des niveaux de rendement entre les adoptants et les non-adoptants. Toutefois cette différence peut être trompeuse car les deux groupes de producteurs peuvent différer de façon systématique, c'est-à-dire que des différences de rendement peuvent exister entre eux bien avant cette adoption. Ainsi, il se dégage un problème de biais de sélection (Heckman, 1997 ; Lee, 2005), ce qui peuvent conduire à des conclusions erronées.

Prenons $A_{i}$ comme une variable dichotomique indiquant le statut du producteur, avec $\mathrm{A}_{\mathrm{i}}=1$ pour les adoptants et $A_{i}=0$ pour les non adoptants. Soient $y_{1 i}$ et $y_{0 i}$, deux (2) valeurs aléatoires du rendement pour un agriculteur i s'il adopte ou non les semences des variétés améliorées du mil. L'impact de

TABLEAU 1. Description des variables du modèle d'adoption du mil amélioré

\begin{tabular}{|c|c|c|}
\hline Variables & Description & Effets attendus \\
\hline \multicolumn{3}{|l|}{ Variable dépendante } \\
\hline Adoption & 1 si oui, 0 si non & \\
\hline \multicolumn{3}{|l|}{ Variables explicatives } \\
\hline Accès village & 1 si le village est facile d'accès, 0 si non & + \\
\hline Sexe & 1 pour les hommes, 0 pour les femmes & \pm \\
\hline Age & L'âge en années des répondants & + \\
\hline Taille & Nombre de personnes du ménage & \pm \\
\hline Education & 1 si instruit, 0 si non & + \\
\hline Vulgarisation & 1 si accès, 0 si non & + \\
\hline Groupement & 1 si membre, 0 si non & + \\
\hline Risque & 1 si l'agriculteur perçoit le risque de production, 0 si non & + \\
\hline Référence & 1 si l'agriculteur préfère la semence améliorée, 0 si non & + \\
\hline Adaptabilité & 1 si l'adaptabilité à la zone de la semence est perçue, 0 si non & + \\
\hline Disponibilité & 1 si la semence améliorée est disponible, 0 si non & + \\
\hline Accessibilité & 1 si la semence améliorée est accessible, 0 si non & + \\
\hline Qualité & 1 si la qualité de la semence améliorée est perçue, 0 si non & + \\
\hline Précocité & 1 si la précocité de la semence améliorée est perçue, 0 si non & + \\
\hline Productivité & 1 si la productivité de semence améliorée est perçue, 0 si non & + \\
\hline
\end{tabular}

$+=$ influence positive attendue $; \pm=$ influence positive ou négative attendue 
l'adoption pour l'agriculteur i est la différence entre les valeurs du rendement avec et sans utilisation des semences $\left(\alpha=y_{1}-y_{0}\right)$. Le problème fondamental à ce niveau est qu'un producteur ne peut être simultanément adoptant et non adoptant (Arouna et Diagne, 2013). Il est donc impossible d'observer à la fois $\mathrm{y}_{1}$ et $\mathrm{y}_{0}$ pour un même agriculteur : c'est le contrefactuelle de Rubin (1974) et l'impossibilité de l'observer représente le problème fondamental de toute évaluation d'impact (Heckman, 2010).

Pour résoudre le problème de ces biais de sélection l'approche non expérimentale est employée. Cette approche est basée sur l'observation et le contrôle des variables qui causent le phénomène de sélection par des méthodes de régression (Heckman, 1997 ; Awotide et al., 2012; Arouna et Diagne, 2013 ;An et Wang, 2016). Plus précisément, c'est la méthode de la variable instrumentale qui est utilisée. La disponibilité des semences est utilisée comme variable instrumentale. A l'aide la fonction Local Average Response Function (LARF), la méthode permet d'estimer le « Local Average Treatment Effect (LATE) qui représente l'impact moyen de l'adoption des semences des variétés améliorées sur le rendement du mil pour la sous-population des adoptants potentiels. L'estimation du LATE se fait à travers des modèles de régression (paramétriques et non paramétriques). Selon Abadie (2003), la fonction LARF a pour équation :

$$
\left.\mathrm{E}\left[\mathrm{g}(\mathrm{y}, \mathrm{A}, \mathrm{x}) \mid \mathrm{A}_{1}=1\right)\right] \frac{1}{\mathrm{P}(\mathrm{A} 1=1)} \mathrm{E}[\mathrm{k} \cdot \mathrm{g}(\mathrm{y}, \mathrm{A}, \mathrm{x})]
$$

$\mathrm{x}$ : variable indépendante, $\mathrm{p}$ : probabilité, $\mathrm{k}$ : poids.

L'estimation de $\mathrm{k}$ se fait à travers la formule suivante :

$$
\mathrm{k}=1-\frac{\mathrm{z}}{\mathrm{P}(\mathrm{z}=1 / \mathrm{x})}
$$

où $\mathrm{z}$ : instrument et $\mathrm{k}$ prend la valeur 1 pour les adoptants potentiels et des valeurs négatives sinon. La probabilité conditionnelle $\mathrm{P}(\mathrm{z}=1 \mid \mathrm{x})$ sera estimée à partir d'un modèle Probit.

A partir de l'équation (4), le Local Average Treatment Effect (LATE) se réduit à :

$\mathrm{E}\left(\mathrm{y} \mid \mathrm{x}, \mathrm{A}_{1}=1\right)=\alpha_{0}+\alpha_{1} \mathrm{~A}+\beta \mathrm{X}+\gamma \mathrm{AX}$

(Arouna et Diagne, 2013)

Avec $\alpha, \beta$ et $\gamma$ sont les vecteurs des paramètres à estimer.

Finalement, le Local Average Treatment Effect (LATE) se réduit à la formule suivante :

LATE $=\alpha_{1}+\gamma \mathrm{X} \quad$ (Arouna et Diagne, 2013)

Les statistiques descriptives ont été réalisées avec le logiciel SPSS (Statistical Package for the Social Sciences) 20. Les estimations du modèle Logit et de LATE ont été faites à l'aide du logiciel STATA (Logiciel statistique de gestion et d'analyse des données) 13.

\section{RESULTATS}

Caractéristiques des producteurs. L'analyse des résultats révèle plusieurs caractéristiques socio-économiques et démographiques des producteurs dont certaines affichent une différence statistiquement significative selon le statut d'adoption de variétés améliorées du mil (Tableau 2). Il ressort de l'analyse que les personnes enquêtées sont majoritairement de sexe masculin $(84,64 \%)$ contre $15,36 \%$ de sexe féminin. Cette situation se justifie du fait qu'en milieu rural nigérien, les exploitations familiales agricoles sont en grande partie dirigées par les hommes. En ce qui concerne l'éducation, un peu plus de la moitié (57\%) de l'échantillon sont instruits. Dans cette zone, trois niveaux d'instruction sont observés à savoir : les scolarisés, les coraniques et les 
TABLEAU 2. Caractéristiques socio-économiques des producteurs selon le statut d'adoption

\begin{tabular}{lccrc}
\hline Variables & $\begin{array}{c}\text { Adoptants } \\
(\mathrm{n}=216)\end{array}$ & $\begin{array}{c}\text { Non-adoptants } \\
(\mathrm{n}=396)\end{array}$ & $\begin{array}{c}\text { Ensemble } \\
(\mathrm{n}=612)\end{array}$ & Différence \\
\hline Femme $(\%)$ & 12,06 & 16,67 & 15,36 & $-4,61^{*}$ \\
Homme (\%) & 87,04 & 83,33 & 84,64 & $3,71^{* *}$ \\
Taux d'éducation (\%) & 67,13 & 51,01 & 57 & $16,12^{* *}$ \\
Accès à la vulgarisation (\%) & 31,48 & 13,89 & 20,10 & $17,59^{* * *}$ \\
Appartenance à une OP (\%) & 25,00 & 11,62 & 16,34 & $13,38^{* *}$ \\
Superficie (ha) & 5,21 & 3,73 & 3,24 & $1,48^{* *}$ \\
Taille du ménage (individus) & 10,26 & 10,61 & 10,49 & $0,35^{\text {ns }}$ \\
Age moyen du chef de ménage (années) & 50,76 & 51,77 & 51,42 & $1,01^{\text {ns }}$ \\
\hline
\end{tabular}

**,* significatif respectivement à $\mathrm{P}<0,01$ et 0,$05 ; \mathrm{ns}=$ non significatif à 0,05

alphabétisés. Pour l'ensemble des producteurs interrogés, l'accès à la vulgarisation agricole et l'appartenance à une organisation des agriculteurs constituent aussi des traits non négligeables $\quad(20,10 \%$ et $16,34 \%$ respectivement). Cependant ces taux restent faibles comparativement aux résultats d'autres études en Afrique (Awotide et al., 2012 ; Yabi et al., 2016 ; Tesfaye et al., 2016). En effet, Awotide et al. (2012) dans leurs travaux sur l'adoption du riz au Nigéria ont trouvé 92\% des hommes, $48 \%$ des instruits et $13 \%$ ont accès à la vulgarisation agricole. Au Bénin, l'étude sur l'adoption des technologies agricoles par Yabi et al. (2016) a rapporté 92\% des hommes, $34 \%$ des instruits et $6 \%$ membres d'une organisation de producteurs. Dans une recherche sur l'adoption du blé en Ethiopie, Tesfaye et al. (2016) ont montré que $36 \%$ de leur échantillon sont instruits. Cette différence est due à la nature des exploitations agricoles enquêtées et des conditions de production plus favorable dans ces pays humides. Un autre constat intéressant est qu'une différence statiquement significative $(\mathrm{P}<0,01)$ est observée entre les adoptants et les non adoptants sur les variables homme, taux d'éducation, accès à la vulgarisation agricole et appartenance à une organisation des producteurs. La différence sur la variable femme entre les adoptants et les non adoptants des variétés améliorées du mil est significative $(\mathrm{P}<0,05)$. La superficie moyenne allouée à la production est de 3,24 ha pour tout l'échantillon. La superficie moyenne des adoptants est significativement $(\mathrm{P}<0,01)$ supérieure à celle des non adoptants. Cette différence pourrait s'expliquer par le niveau de richesse des producteurs dont la mise en valeur de grande superficie possédée est un facteur en milieu rural. En effet des études ont montré que la richesse est un facteur déterminant l'adoption des technologies agricoles (Roussy et al., 2015). Le nombre moyen de personnes que compte une exploitation agricole familiale est de 10 personnes. Elle est supérieure à la moyenne nationale qui est de 7 personnes. Au moment de l'enquête, l'âge moyen des personnes interviewées est de 51 ans.

Déterminants de l'adoption des variétés améliorées du mil. Les résultats de l'estimation du modèle Logit d'adoption des semences des variétés améliorées du mil sont consignés dans le Tableau 3. Le test de ratio de vraisemblance indique que le modèle estimé est globalement significatif $(\mathrm{P}<0,001)$, ce qui montre la bonne spécification du modèle et que les variables retenues permettent d'expliquer significativement l'adoption. Le taux d'adoption de ces semences est de 35,29\%. Cette adoption est significativement déterminée à $\mathrm{P}<0,001$ par la perception de risque de production, l'accès à la vulgarisation, la disponibilité et la productivité ; à $\mathrm{P}<0,01$ par 
TABLEAU 3. Estimation du modèle Logit d'adoption des semences améliorées du niébé

\begin{tabular}{|c|c|c|c|}
\hline Variables & Coefficients & Significativité & Effets marginaux \\
\hline Accessibilité village & 0,269 & $0,446^{\mathrm{ns}}$ & 0,025 \\
\hline Sexe & $-0,123$ & $0,799^{\text {ns }}$ & $-0,110$ \\
\hline Age & $-0,304$ & $0,001^{* *}$ & $-0,028$ \\
\hline Taille & $-0,061$ & $0,023^{*}$ & $-0,006$ \\
\hline Education & 0,815 & $0,004^{* *}$ & 0,076 \\
\hline Vulgarisation & 1,905 & $0,000^{* * *}$ & 0,166 \\
\hline Groupement & 0,294 & $0,481^{\mathrm{ns}}$ & 0,026 \\
\hline Risque & 1,986 & $0,000^{* * *}$ & 0,215 \\
\hline Préférence & 0,997 & $0,157^{\mathrm{ns}}$ & 0,099 \\
\hline Adaptabilité & 2,063 & $0,001^{* *}$ & 0,224 \\
\hline Disponibilité & 2,093 & $0,000^{* * *}$ & 0,218 \\
\hline Accessibilité & $-0,642$ & $0,155^{\mathrm{ns}}$ & $-0,055$ \\
\hline Qualité & $-0,755$ & $0,265^{\mathrm{ns}}$ & $-0,062$ \\
\hline Précocité & 1,146 & $0,003^{* *}$ & 0,098 \\
\hline Productivité & 1,418 & $0,000^{* * *}$ & 0,144 \\
\hline Constante & 1,634 & $0,470^{\mathrm{ns}}$ & \\
\hline Nombre d'observations & & \multicolumn{2}{|c|}{612} \\
\hline Log de Vraisemblance & & \multicolumn{2}{|c|}{$-185,500$} \\
\hline Pseudo $\mathrm{R}^{2}$ & & \multicolumn{2}{|c|}{0,558} \\
\hline LR test & & \multicolumn{2}{|r|}{$468,46 * * *$} \\
\hline Proportion d'adoptants de semence améliorée du niébe & & \multicolumn{2}{|r|}{$35,29 \%$} \\
\hline
\end{tabular}

******,* significatif respectivement à $\mathrm{P}<0,001 ; 0,01$ et 0,$05 ; \mathrm{ns}=$ non significatif à 0,05

l'adaptabilité, la précocité, l'âge, l'éducation et à $\mathrm{P}<0,05$ par la taille du ménage. Les degrés d'expression de ces variables ainsi que leur signe sont présentés dans la deuxième colonne du Tableau 3.

Les effets marginaux présentés sur la dernière colonne du Tableau 3 montrent l'impact de la variation d'une unité de chaque variable sur la probabilité d'adoption. Ainsi, la variation d'une unité de l'éducation, la perception de risque de production, l'accès à la vulgarisation ; la disponibilité, l'adaptabilité et la productivité des semences des variétés améliorées et la taille de l'exploitation augmente la probabilité d'adoption respectivement de 0,$08 ; 0,22 ; 0,17 ; 0,22 ; 0,22$ et 0,14 . Cette variation d'une unité de l'âge, la taille de l'exploitation et la précocité des semences entraine une diminution de la probabilité d'adoption respectivement de $0,03,0,01$ et 0,09 .
Impact de l'adoption des variétés améliorées sur le rendement du mil. Pour estimer l'impact de l'utilisation des variétés améliorées sur la productivité du mil, nous utilisons la méthode de Local Average Response Function (LARF). Nous considérons le rendement des agriculteurs par hectare comme variable de résultat, le statut d'adoption, les variables indépendantes et la disponibilité des semences comme variable instrumentale. Les résultats de l'estimation sont présentés dans le Tableau 4. L'analyse de la comparaison de moyenne montre une différence positive de $211,74 \mathrm{~kg} \mathrm{ha}^{-1}$ de rendement entre les agriculteurs qui utilisent les variétés améliorées du mil et leurs homologues qui ne les utilisent pas. Cette différence est statistiquement significative $(\mathrm{P}<0,001)$ entre ces deux groupes. Les résultats du LATE indiquent aussi que l'adoption de ces semences permet 
TABLEAU 4. Résultats d'estimation de l'impact des semences améliorées sur le rendement du mil (LARF)

\begin{tabular}{|c|c|c|c|c|}
\hline Estimation & Paramètres & Erreur Standard & Valeur-Z & P ÂlZI \\
\hline \multicolumn{5}{|l|}{ Méthode naïve } \\
\hline Différence & $211,74^{* * *}$ & 8,29 & $-4,74$ & 0,000 \\
\hline Adoptants & $497,87^{* * *}$ & 19,49 & 6,23 & 0,000 \\
\hline Non-adoptants & $286,07^{* * *}$ & 14,21 & 3,46 & 0,000 \\
\hline \multicolumn{5}{|c|}{ Méthode de Local Average Response Function (LARF) } \\
\hline LATE & $406,93^{* * *}$ & 47,81 & 8,51 & 0,000 \\
\hline
\end{tabular}

${ }^{* * * *}=$ Significatif à $\mathrm{P}<0,001$

d'augmenter significativement $(\mathrm{P}<0,001)$ la productivité du mil chez les adoptants potentiels comme le montre la valeur estimée du LATE. En effet, l'adoption des semences des variétés améliorées du mil permet aux agriculteurs potentiels d'augmenter le rendement de 406,93 $\mathrm{kg} \mathrm{ha}^{-1}$. Cela pourrait être interprété comme le résultat du changement technique occasionné par les semences. Ces résultats confirment l'hypothèse selon laquelle les producteurs du mil améliorent leur production en adoptant des nouvelles. Cette augmentation pourrait avoir un effet positif sur la sécurité alimentaire des ménages et par conséquent sur leur bien-être. L'adoption de ces semences est donc favorable aux pauvres, elle donne ainsi des preuves empiriques aux décideurs politiques agricoles leur encourageant dans la diffusion des semences des variétés améliorées au Niger.

Impact de la production du mil amélioré sur la durée de la consommation alimentaire. Une analyse de la perception des agriculteurs sur le nombre de mois de consommation que peut couvrir leur production du mil a été faite. Les résultats de cette analyse indiquent que la production du mil permet de couvrir en moyenne 5,21 mois contre 2,75 mois de consommation respectivement chez les adoptants et les non adoptants. L'analyse de la comparaison de moyenne montre donc une différence positive de 2,46 mois de consommation entre les agriculteurs qui utilisent les semences des variétés améliorées du mil et leurs homologues non utilisateurs. Cette différence est statistiquement significative $(\mathrm{P}<0,05)$. Ainsi, en augmentant la production, les semences des variétés améliorées accroissent la sécurité alimentaire des ménages. Ces types de semences constituent donc une alternative pour améliorer les conditions de vie des populations.

\section{DISCUSSION}

Déterminants de l'adoption des variétés améliorées du mil. Les producteurs les plus âgés (expérimentés) sont moins susceptibles ou ont tendance à rejeter les variétés améliorées du mil contrairement aux jeunes agriculteurs. Ce résultat montre que les personnes âgées ne sont pas favorables au changement des variétés proposées par la vulgarisation, probablement pour des raisons culturelles ou de réticence. On peut aussi penser que les producteurs les plus âgés valorisent moins les bénéfices à long terme des améliorées. Cependant, ce résultat sur l'influence de l'âge est contraire à la littérature (Ngondjeb et al., 2014 ; Yabi et al., 2016). En effet, ces auteurs ont montré que les agriculteurs les plus âgés adopteraient plus facilement les innovations que les jeunes agriculteurs. La taille du ménage a une influence négative sur l'adoption des semences. Ce résultat corrobore les conclusions de Folefack et al. (2012) qui ont 
trouvé que la taille du ménage a un effet significatif et négatif sur la probabilité du choix de la stratégie de fumure organique. Le rôle positif de l'éducation et la vulgarisation a été révélé par la littérature (Ngondjeb et al., 2014 ; Roussy et al., 2015 ; Yabi et al., 2016). Les agriculteurs les plus éduqués disposent de plus d'informations leur permettant de mieux évaluer les variétés améliorées et ainsi leur niveau d'incertitude est limité. Plus les producteurs sont instruits, plus ils sont ouverts aux innovations technologiques. Le contact des producteurs avec les agents de la vulgarisation leur permettent d'accéder à l'information et à la formation sur les nouvelles technologies. La perception sur les caractéristiques des semences comme la disponibilité, l'adaptabilité, la précocité et la productivité accroisse l'adoption de ces semences. De tels résultats confirment les études de Abdulai et Huffman (2014) qui ont abouti à la conclusion selon laquelle les perceptions d'une innovation se font à travers ses caractéristiques telles que la profitabilité, l'efficacité et l'adaptabilité. Plus les agriculteurs perçoivent l'intérêt de l'innovation, plus ils adoptent. Les perceptions des agriculteurs sur l'innovation sont fortement liées aux perceptions de risques et peuvent être modifiées par l'acquisition d'expériences ou d'informations. Les agriculteurs qui perçoivent le risque de production sont plus susceptibles d'adopter les semences des variétés améliorées du mil, ce qui rejoint les travaux de Yegbemey et al. (2014) qui ont montré que les producteurs de maïs qui perçoivent les changements climatiques utilisent les semences améliorées pour s'adapter. Enfin, l'appartenance à un groupement de producteur n'a aucune influence sur l'adoption. Cette situation est inattendue mais montre que les organisations de producteurs des villages d'étude ne fournissent pas d'informations sur les semences améliorées à leurs membres. Ces structures paysannes n'existent que sur le papier mais n'exercent aucune activité puisqu'elles ont été mises en place à la demande des partenaires au développement et non à l'initiative propre des agriculteurs. Ce résultat est en contradiction avec ceux de Ngondjeb et al. (2014). En effet, cet auteur a trouvé que les réseaux locaux et la coopération entre les agriculteurs favorisent l'adoption des technologies agricoles grâce aux échanges d'informations et de partage d'expériences. Impact de l'adoption des variétés améliorées sur le rendement du mil

Les résultats sur l'analyse de la comparaison de moyenne et du LATE ont tous montré une différence positive et statistiquement significative $(P<0,001)$ entre les agriculteurs qui utilisent les variétés améliorées du mil et leurs homologues qui ne les utilisent pas. La valeur estimée du LATE est de 406,93 $\mathrm{kg} \mathrm{ha}^{-1}$. Cela pourrait être interprété comme le résultat du changement technique occasionné par l'adoption des variétés améliorées. Ces résultats confirment l'hypothèse selon laquelle les producteurs du mil et du niébé améliorent leur production en adoptant des nouvelles technologies agricoles. Ces résultats rejoignent ceux de nombreuses études d'impact qui ont trouvé que l'adoption des semences améliorées augmente significativement le rendement des cultures (Nouhoheflim et al., 2004 ; Assiya, 2010 ; Awotide et al., 2012 ; FAO, 2013 ; Maruod et al., 2013 ; Arouna et Diagne, 2013 ; Meughoyi, 2015 ; Ogunniyi et Kehinde, 2015 ; Tesfaye et al., 2016). Au Bénin, Nouhoheflim et al. (2004) ont trouvé que l'utilisation des variétés améliorées du niébé a induit une différence significative sur le rendement entre les adoptions et les non adoptants. Au Niger, Assiya (2010) a conclu que l'adoption des variétés améliorées du mil et du niébé permet d'augmenter le rendement des agriculteurs. Au Nigéria, Awotide et al. (2012) ont rapporté que l'adoption des variétés améliorées du riz a un impact positif et significatif sur la productivité $\left(358,89 \mathrm{~kg} \mathrm{ha}^{-1}\right)$. De son côté, FAO (2013) précise une augmentation de plus de $25 \%$ de rendement peut être obtenu sir les producteurs du Niger utilisent les variétés améliorées du mil et du niébé. Au Soudan, Maruod et al. 
(2013) ont constaté que plus de $200 \%$ de productivité peuvent être enregistré par les agriculteurs qui adoptent les nouvelles variétés d'arachide. Au Bénin, Arouna et Diagne (2013) ont montré que la multiplication de semences des variétés améliorées permet aux riziculteurs d'augmenter leur rendement en riz de $1924 \mathrm{~kg}$ ha $^{-1}$. Les conclusions de Meughoyi (2015) au Cameroun ont révélé que les producteurs de maïs qui adoptent les nouvelles variétés produisent 1,42 fois plus que ceux qui n'en adoptent pas. Dans leur étude au Nigéria, Ogunniyi et Kehinde (2015) ont trouvé une augmentation de rendement de $120,95 \mathrm{~kg}$ ha $^{-1}$ chez les agriculteurs qui adoptent les variétés améliorées du maïs. Enfin, Tesfaye $e t$ al. (2016) en Ethiopie ont mis en exergue une augmentation 1 à $1,1 \mathrm{t}^{-1} \mathrm{a}^{-1}$ peut être obtenue si les producteurs de blé utilisant des nouvelles variétés issue de la recherche agronomique.

Impact de la production du mil amélioré sur la durée de la consommation alimentaire. La portion de la production du mil permet de couvrir en moyenne 5,21 mois contre 2,75 mois de consommation respectivement chez les adoptants et les non adoptants. Chez les ménages adoptants, une prolongation de la disponibilité alimentaire de l'ordre deux mois environ est induite par l'utilisation des variétés améliorées du mil. Ainsi, en augmentant la production, les semences des variétés améliorées du mil accroissent la sécurité alimentaire des ménages. De tels résultats sur l'impact des variétés améliorées sur la sécurité alimentaire ont été relevés dans la littérature (FAO, 2013 ; Schiferaw et al., 2014). Au Niger, FAO (2013) a trouvé que les agriculteurs qui utilisent les variétés améliorées du mil et du niébé peuvent consommer leur production durant cinq (5) mois alors que leurs homologues non utilisateurs ne peuvent consommer leur production que durant trois mois (3). En Ethiopie, Schiferaw et al. (2014) ont conclu que l'adoption des nouvelles variétés du blé accroît la sécurité alimentaire des ménages.

\section{CONCLUSION}

Les différentes analyses mettent en évidence les déterminants et l'impact de l'adoption des semences des variétés améliorées sur la productivité du mil au Niger. Le modèle Logit a permis d'identifier les facteurs influençant l'adoption. L'étude a révélé que le taux d'adoption de ces variétés est de 35,29\%. Cette adoption est déterminée par des variables comme l'âge, la taille du ménage, le niveau d'éducation, l'accès à la vulgarisation agricole et la perception sur le risque de production et les caractéristiques des semences (disponibilité, adaptabilité, précocité et productivité). Elle montre aussi que les adoptants différent des non adoptants en terme des caractéristiques comme le sexe, le niveau d'instruction, l'accès à la vulgarisation, l'appartenance à une organisation des producteurs et les superficies. Les semences ont permis d'augmenter significativement la productivité du mil chez les adoptants potentiels. Ainsi, elles pourraient constituer un instrument important de politiques agricoles visant la sécurité alimentaire et la durabilité de la production. Il devient donc urgent à ce que les décideurs puissent intensifier les actions de vulgarisation des semences des variétés améliorées en milieu rural. Dans ce sens, les déterminants de l'adoption identifiés sont à ne pas négliger. Une des lacunes de l'étude est de ne pas faire de distinction entre les différences variétés adoptées pour déceler l'impact réel de chacune d'entre elles.

\section{REMERCIEMENTS}

Les auteurs remercient l'ONG Comité d'Appui au Développement Local (CADEL) et le Programme de Productivité Agricole en Afrique de l'Ouest (PPAAO) pour le soutien financier. Nous remercions également les personnes enquêtées pour les données fournies et les évaluateurs pour leurs suggestions d'amélioration du manuscrit. 


\section{REFERENCES}

Abadie, A. 2003. Semi-parametric instrumental variable estimation of treatment response Models. Journal of Econometrics 113: 231-263.

Abdulai, A. et Huffman, W.E. 2014. The adoption and impact of soil and water conservation technology: An endogenous switching regression application. Land Economics 90: 26-43.

AGRHYMET. 2012. Le Sahel face aux changements climatiques : Enjeux pour un développement durable; Niamey-Niger. 38pp.

An, W. et Wang, X. 2016. Instrumental variable estimation of causal effects through local Aaverage response functions. Journal of Statiscal Software 71 (1) : 1-13.

Arouna, A. et Diagne, A. 2013. Impact de la production de semence riz sur le rendement et le revenu des ménages agricoles: une étude de cas du Bénin. $4^{\text {th }}$ International Conference of the African Association of Agricultural Economists. Hammamet, Tunisia.

Assiya, A.C. 2010. Evaluation des impacts socio-économiques de l'utilisation des variétés améliorées (Mil HKP et Niébé TN578) au niveau ménages dans la région de Zinder : cas de Garagoumsa, Tirmini et Tanout au Niger. Mémoire d'Ingénieur Agronome. Institut Polytechnique Rural, Mali. 107pp.

Awotide, B., Diagne, A. et Omonona, B. 2012. Impact of improved agricultural technology adoption on sustainable rice productivity and rural farmers' welfare in Nigeria: A local average treatment effect (Late) technique. African Economic Conference. Kigali, Rwanda.

Banque Mondiale. 2010. Rapport annuel : bilan de l'exercice. 37pp. http://siteresources. worldbank.org/EXTANNREP2010FRE/ Resources/Banquemondiale Rapportannuel2010.pdf Consulté le 22 décembre 2016.
Fall, A. A. 2006. Impact du crédit sur le revenu des riziculteurs de la vallée du fleuve Sénégal. Thèse de doctorat, université Montpellier I, France. 357pp.

Folefack, D.P., Sale, A. et Wakponou, A. 2012. Facteurs affectant l'utilisation de la fumure organique dans les exploitations agricoles en zone sahélienne du Cameroun. Afrique Science: Revue Internationale des Sciences et Technologie 8 (2): 22-33.

Heckman, J. 2010. Building bridges between structural and program evaluation approaches to evaluating policy. Journal of Economic Literature 48 (2) : 356-398.

Heckman, J. 1997. Instrumental variables: A study of the implicit assumptions underlying one widely used Estimator for Program Evaluations. Journal of Human Resources $32: 441-462$.

Institut National de la Statistique (INS) et Système d'Alerte Précoce et de prévention des Catastrophes (SAP/PC). 2016. Enquête conjointe sur la vulnérabilité à l'insécurité alimentaire des ménages au Niger. 168pp. http://documents.wfp.org/stellent/groups/ public/documents/ena/wfp286674. pdf?iframe (consulté le 16/12/2016).

Lee M.J. 2005. Micro-Econometrics for policy, program and treatment effects. Advanced Texts in Econometrics. Oxford University Press.

Maruod, E. Maruod, Elkhalil E. Breima, Elrasheid E. Elkhidir and Ahmed M. El Naim. 2013. Impact of improved seeds on small farmers productivity, income and livelihood in Umruwaba Locality of North Kordofan, Sudan. International Journal of Agriculture and Forestry 3(6) : 203-208.

Meughoyi, T.C. 2015. Semences améliorées et productivité agricole des exploitations familiales agricoles au Cameroun. Conférence sur «Accéléré la productivité agricole: la technologie et l'innovation, les actifs, l'accès au financement » au Cameroun, pp. 1-13. 
Mbétid-Bessane, E. 2014. Adoption et intensification du nouveau riz pour l'Afrique en Centrafrique. Tropicultura 32 (1): 1621.

Ministère du développement Agricole. 2016. Annuaire national de disponibilité en semences améliorées au Niger. 90pp. http:/ /www.reca-niger.org/spip.php?article730 (consulté le 09/09/2016).

Ministère du Développement Agricole du Niger. 2012. La politique semencière nationale. 45pp. www.faolex.fao.org/docs/pdf/ ner144905.pdf (consulté le 04/01/2016).

Institut de Recherche pour le développement (IRD). 2004. Ressources génétiques des mil en Afrique de l'Ouest. 192pp. DOI : 10.4000/books.irdeditions.511 http:// books.openedition.org/irdeditions/511 Consulté le 22 décembre 2016.

Moti, J., Menale, K. et Paswel, M. 2015. Impact of improved maize variety adoption on household food security in Ethiopia: An endogenous switching regression approach. Agriculture in an interconnected World. Milan, Italy: International Conference of Agricultural Economics.

Ngondjeb, Y., Nje, P. et Havar, M. 2011. Déterminants de l'adoption des techniques de lutte contre l'érosion hydrique en zone cotonnière du Cameroun. Revue d'élevage et de médecine vétérinaire des pays tropicaux 64 (1): 9-19.

Ngendjeb, Y.D., Kamgnia, D.B., Nje, P. et Havard, M. 2014. L'évaluation économique de l'investissement dans la conservation des sols : le cas des aménagements antiérosifs dans le bassin versant du lac Logdo au Cameroun. Canadian Journal of Agricultural Economics 62 : 393-410.

Nouhoheflin, T., Coulibaly, O. et Adegbidi, A. 2002. Impact des nouvelles technologies de culture du niébé sur la production, les revenus et leur distribution au Bénin in Savanes africaines: Des espaces en mutation, des acteurs face à de nouveaux défis. Actes du colloque, Garoua, Cameroun Cirad, Montpellier, France, 6 p.
Ogunniyi, A. et Kehinde, O. 2015. Impact of agricultural innovation on improved livelihood and productivity outcomes among smallholder farmers in rural Nigeria. Maastricht School of Management, Working paper $\mathrm{N}^{\circ} 2015 / 07$, pp. 1-23.

Organisation des Nations Unies pour l'Alimentation et l'Agriculture (FAO). 2016. La situation Mondiale de l'alimentation et de l'agriculture : changement climatique, agriculture et sécurité alimentaire. 234pp. http://www.fao.org/publications/card/fr/c/ 3fe586ed-52e8-47d4-a2e4-1b4282f9a5d2/ Consulté le 12 décembre 2016.

Organisation des Nations Unies pour l'Alimentation et l'Agriculture (FAO). 2015. L'état de l'insécurité alimentaire dans le monde. 66pp. http://www.fao.org/3/ai4646f.pdf Consulté le 12 décembre 2016.

Organisation des Nations Unies pour l'Alimentation et l'Agriculture (FAO)Niger. 2013. Multiplication et diffusion des semences de qualité des variétés améliorées et adaptées au changement climatique. Fiche de bonne pratique, $8 \mathrm{p}$. http:// www.fao.org/docrep/019/ar725f/ ar725f.pdf (consulté le 14/08/2016).

Polome, P. 2013. Econométrie II. https:// www-perso.gate.cnrs.fr/polome/.../ Ectx II L3 ch0 2012-13.pdf consulté le 7 septembre 2016.

Roussy, C., Ridier, A. et Chaib, K. 2015. Adoption d'innovations par les agriculteurs : rôle des perceptions et des préférences. Working Paper SMARTLERECO N ${ }^{\circ}$ 15-03. https://www6.rennes. inra.fr/smart/content/download/.../WP1503.pdf consulté le 21 octobre 2015.

Rubin, D. 1974. Estimating causal effects of treatments in randomized and nonrandomized studies. Journal of Educational Psychology 66 : 688-701.

Shiferaw, B., Menale, K., Moti, J. et Chilot, Y. 2014. Adoption of improved wheat varieties and impacts on household food security in Ethiopia. Food Policy (Elsevier Ltd.) 44: 272-284. 
Tesfaye, B., Bedada, B. and Mesay, Y. 2016. Impact of improved wheat technology adoption on productivity and income in Ethiopia. African Crop Science Journal 24 (Issue supplement S1) : 127-135.

Yabi, J.A., Bachabi, F.X., Labiyi, I.A., Ode, C.A. et Ayena, R.L. 2016. Déterminants socio-économiques de l'adoption des pratiques culturales de gestion de la fetilité des sols utilisées dans la commune de Ouaké au Nord-Ouest du Bénin. Int. J. Bio. Chem. Sci 10 (2) : 779-792.

Yegbemey, R.N., Yabi, J.A., Aihounton, G.B. et Paraiso. A. 2014. Modélisation simultanée de la perception et de l'adaptation au changement climatique : cas des producteurs de maïs du Nord Bénin (Afrique de l'Ouest). Cahier d'Agriculture 23 (3) : 177-187. 